\title{
Virtual and Physical Prototyping to Demonstrate Design Feasibility
}

\author{
Steven M. DeMers \\ Graduate Student \\ University of Waterloo \\ sdemers@uwaterloo.ca
}

\author{
Stephan B. Lambert \\ Professor \\ University of Waterloo \\ steve@uwaterloo.ca
}

\begin{abstract}
Hybrid electric vehicle technology has become a preferred method for the automotive industry to reduce environmental impact and fuel consumption of their vehicles. However, the added cost of the hybrid electric system has hindered their sales. A more cost effective design of an electro-hydraulic braking system is presented. During the design process, emphasis was placed on demonstrating conceptual and system feasibility using virtual and physical prototyping. The role of prototyping in the design process is presented and discussed.
\end{abstract}

\section{Introduction}

With rising gas prices and the overwhelming concern for the environment, consumers and the government have forced the automotive industry to start producing more fuel efficient vehicles with less environmental impact. One promising method that is currently being implemented is the hybrid electric vehicle (HEV).

Hybrid vehicles are defined as vehicles that have two or more power sources [1]. There are a large number of possible variations but the most common layout of hybrid vehicles today combines an internal combustion engine (ICE) with an electric motor and energy storage system (ESS) [2].

HEV's have the distinct advantage of regenerative braking. The electric motor, normally used for propulsion, can be used as a generator to convert kinetic energy of the vehicle back into electrical energy during braking, rather than wasting energy as heat. This electrical energy can then be stored in an ESS (e.g. batteries or ultracapacitors) and later released to propel the vehicle using the electric motor.

For regenerative braking to operate safely and effectively, electrical and mechanical braking must be fully integrated. This requires that the combination of regenerative and mechanical braking be controlled smoothly and accurately to meet driver demand.

Controlling regenerative braking torque can be read- ily accomplished using control algorithms for electric motors. However, controlling the mechanical braking torque independently of the driver pedal force, while maintaining proper safety back-ups, is more of a challenge. To overcome this problem, a system was developed that would electro-mechanically attenuate the pressure in the brake booster in order to control the amount of mechanical torque developed by the braking system.

To develop and verify this system, a systematic design approach was utilized with an emphasis on demonstrating design feasibility using a combination of virtual and physical prototyping. The prototypes were then used to optimize and tune the system in a cost and time efficient manner.

\section{Design Methodology}

Before the design for a project can begin, the need for that product must be established to identify and market research-specific deliverables to meet customer demands [3]. The results of the project definition phase are then used to focus the generation and evaluation of conceptual designs. During the conceptual design phase, designs will be generated and evaluated iteratively based on the project definition with the goal of choosing the best design.

Once a concept has been chosen, it is embodied during the preliminary design phase and continuously refined with the aid of virtual and physical prototypes until a demonstratively working system is developed.

\subsection{Project Definition}

Although HEV's have improved emissions and fuel economy over traditional automobiles, the added cost of the hybrid system deters some customers from purchasing these vehicles [4]. One of the more expensive systems added to HEV's is the electro-hydraulic braking (EHB) system [5].

As part of a larger research programme to investigate the application of ultracapacitors for regenerative braking [6], a low-cost approach to the integration of 
mechanical and regenerative braking was pursued. The new hybrid brake system must integrate regenerative and mechanical braking in a manner that is transparent to the driver, and maximize regenerative braking potential. Ideally, mechanical braking should vary from $0 \%$ to $100 \%$ of demand, independent of the pedal force. It must also have an adequate response time to address driver demand and moment to moment variations in regenerative braking. A maximum response time of 0.6 seconds is suggested from regulations [7].

Figure 1 represents a typical braking event considered for the project. The driver demand curve is created by the force exerted on the brake pedal and is directly proportional to requested torque. As time elapses, the angular speed of the electric motor will slow, which will reduce available regenerative braking. The total required brake force must be supplemented by the mechanical braking system. At some point full mechanical braking will be required. It was decided that a reasonable time response $(\Delta t)$ to go from maximum to full mechanical braking would be less than 0.4 seconds.

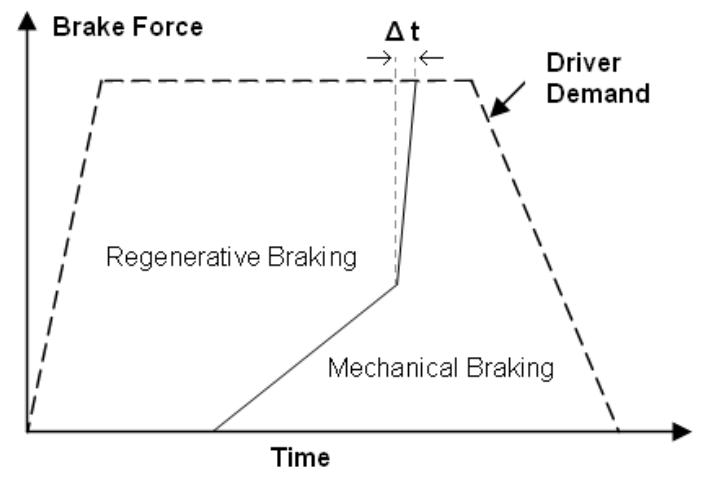

Figure 1: Typical distribution of regenerative and mechanical braking force required

\subsection{Conceptual Design}

During the conceptual design phase, existing and conceptual designs were considered in order to choose a design that best fits the project definition.

The current EHB system in most HEV's (e.g. Toyota Prius) uses a brake pedal simulator, which is separate from the hydraulic braking circuit, to establish driver braking demand. The braking demand is then sent to a system that contains a high pressure hydraulic pump, accumulator and proportional control valves. The proportional control valves allow the brake line fluid to flow to each wheel at predefined pressures determined by the braking demand.

The simplest alternate concept considered involved downsizing a standard automotive braking system and supplementing the extra braking torque with regenerative braking. This system required no major modifications to the existing brake system and the regenerative braking could easily be controlled using simple algorithms. However, this does not achieve the desired operational flexibility.

The second concept was an electro-mechanical brake system that used small electric motors in the brake caliper to push the brake pads against the brake rotor to create braking torque. This eliminates the hydraulic system from the standard braking system. However, a hydraulic back-up system would still need to be installed in case of primary system failure. This system requires a brake pedal simulator as a method of establishing braking demand.

The last concept was a new electro-mechanical brake booster design (Figure 2, not currently used in the automotive industry. The idea for this concept was first suggested by Chris Mendes, a former master's student at the University of Waterloo. A similar concept was found following a patent search [8]. The concept was refined by the first author. It operates by using two electro-mechanical valves to attenuate the boost, and hence braking force, created by the brake booster.

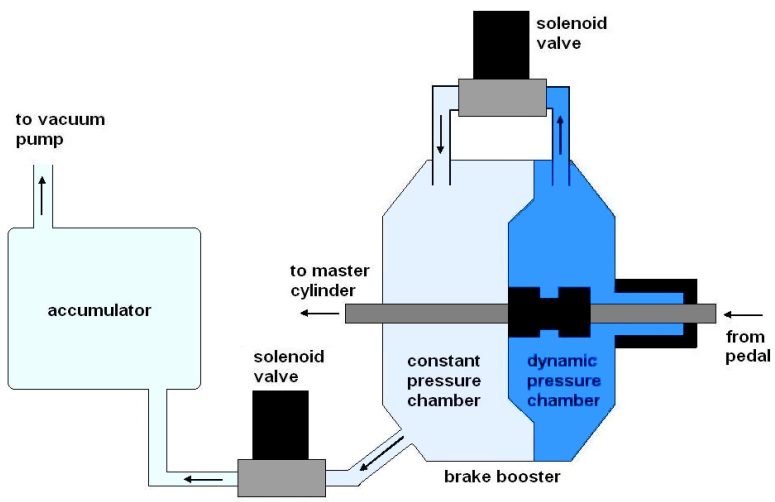

Figure 2: Electro-mechanical brake booster concept

After careful consideration, the electro-mechanical brake booster was chosen. The concept uses an inexpensive, low pressure pneumatic system that can be easily sourced, it requires no extra back-up systems in case of failure, and has the best potential to meet all the requirements.

In standard automotive brake boosters, the vacuum created in the intake manifold is used to create a vacuum in both chambers of the brake booster. When the driver applies the brake, air enters the dynamic pressure chamber of the brake booster according to the force exerted on the brake pedal. This results in a pressure difference between the two chambers which creates an added boost 
to the driver's pedal force.

The proposed system electro-mechanically controls the pressure differential created within the brake booster using two valves. The first valve allows atmospheric air to enter the brake booster, which will reduce the vacuum created and the effective mechanical braking torque. The second valve allows air to leave the brake booster using a vacuum accumulator, which will increase the boost created. Solenoid valves were chosen because of their low cost and high reliability. Even though they cannot infinitely vary the flow like a proportional control valve, the discrete on and off stages of the solenoid valves should be small enough that they are not noticeable by the driver. A schematic of the electromechanical brake booster can be seen in Figure 3

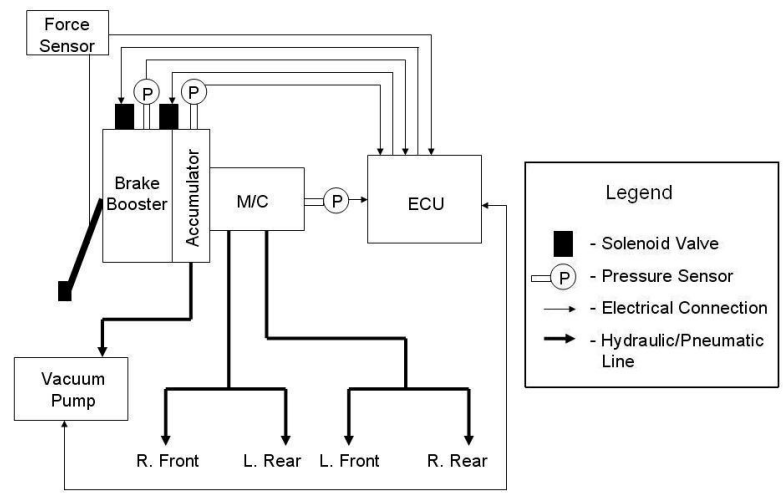

Figure 3: Electro-mechanical brake booster schematic

\subsubsection{Proof of Conceptual Design}

Since standard automotive brake boosters use a variable pressure differential to boost pedal force, it was assumed that the same principle could be used for the electromechanical brake concept. However, it had to be verified that the electro-mechanical valves could provide an adequate response time. To evaluate the time response, a simple virtual prototype was created that modelled an estimated brake booster volume of 2.5 litres, an infinite accumulator held at $30 \mathrm{kPa}$, and a valve with a flow factor of 1.6. It was decided that reducing the pressure in the brake booster from atmospheric pressure $(101.3 \mathrm{kPa})$ to $75 \mathrm{kPa}$ would correspond to full mechanical braking. This virtual test was then carried out physically using a brake booster volume of 2.5 litres, a 4 litre accumulator initially at $30 \mathrm{kPa}$, and a valve with a flow factor of 1.63 .

The results from these tests can be seen in Figure 4 A 10 second test was run in which the valves were activated after 8 seconds and were closed after the pressure dropped below $75 \mathrm{kPa}$. The figure is enlarged around the region of the graph that shows the response time of the system. It is shown that both the simple virtual and physical prototypes have response times less than 0.4 seconds to demonstrate the feasibility of the concept.

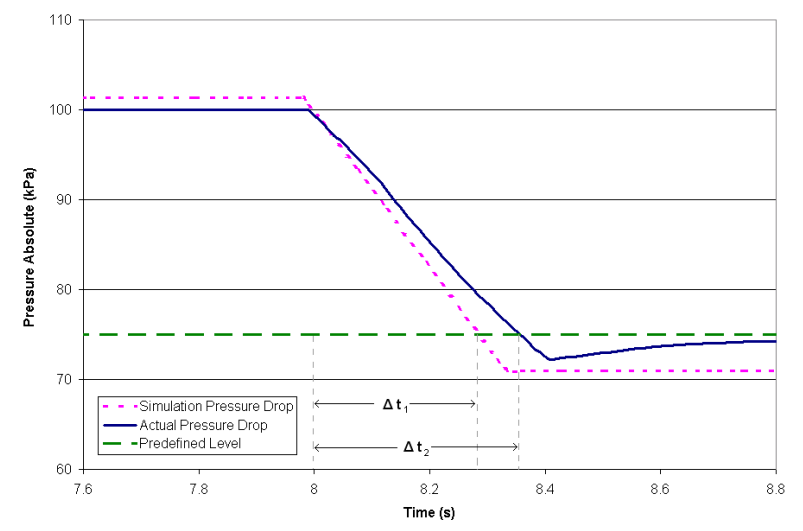

Figure 4: Virtual and physical response time test

\section{Preliminary Design}

The focus of the preliminary design phase is to demonstrate the ability of the system to function as required. It was decided that both virtual and physical mechani$\mathrm{cal} /$ regenerative braking prototypes would be created to demonstrate this function. The system used a flywheel to simulate the inertia of the vehicle, which was connected to a three phase AC induction motor with suitable power electronics, vector control and an ESS to allow for regenerative braking. A standard automotive braking system was added to the testbench, which included the brake pedal, brake booster and master cylinder. The brake booster was then modified to implement the electro-mechanical brake booster and electromechanical valves were added. The testbench is shown in Figure 5. Although there are many components associated with the prototype system, the focus of this discussion is the electro-mechanical brake booster.

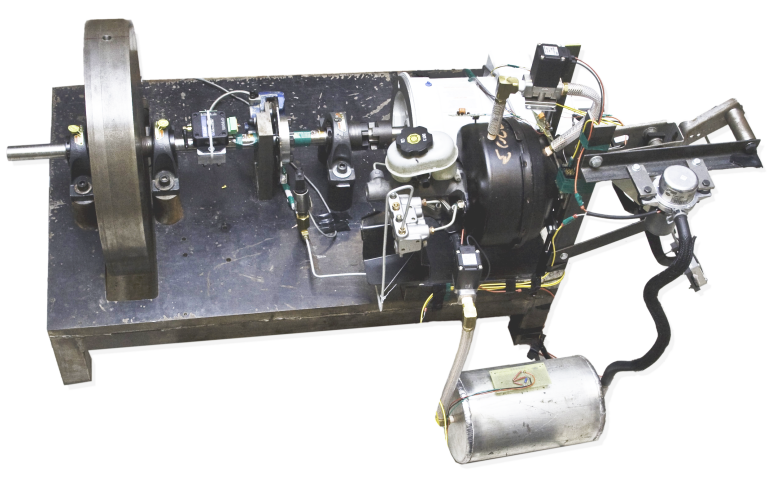

Figure 5: Prototype of system 


\subsection{Virtual Prototyping}

To analyze the system, a model of the mechani$\mathrm{cal} /$ regenerative braking testbench including the electromechanical brake booster was created in Matlab 7.3.0 (R2006b). A schematic of this system can be seen in Figure 6 .

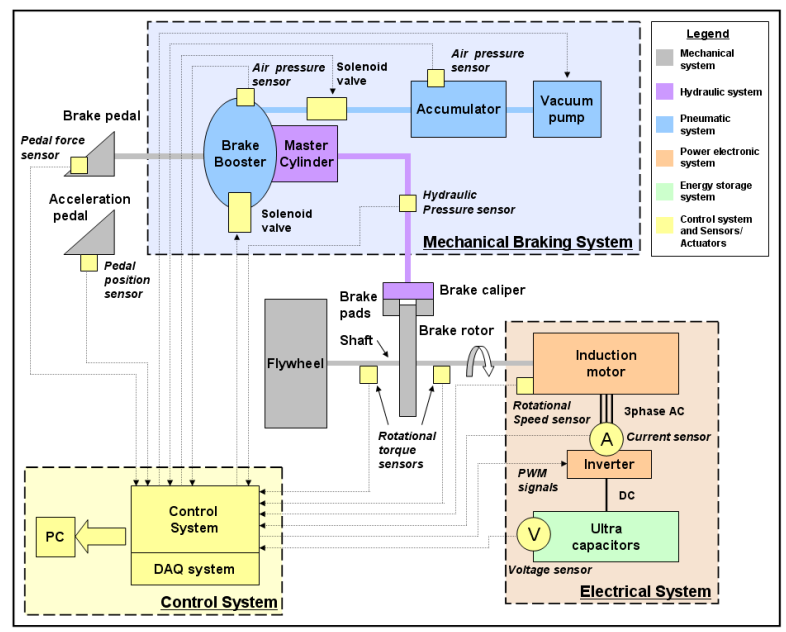

Figure 6: Simulation overview schematic

The goal for this simulation of the mechanical brake system was to develop reduced-order models of the concept that could be used to evaluate the feasibility of the design. These models could then be used to optimize the system. The model was based on a conventional brake system [9], and modified to simulate the electromechanical brake booster. For ease of calculation, the brake booster was modelled using air chamber thermodynamics for the constant and dynamic chambers. The air in the brake booster was considered to be an ideal gas undergoing isothermal expansion or compression, which results in the pressure in each chamber being related to the mass of air in the chamber and diaphragm motion.

The solenoid valves used to attenuate the pressure in the constant pressure chamber of the brake booster were modelled using flow factors from current market solenoid valves [10], and first order lag characteristics were used to simulate the valve opening and closing reaction time. To control the solenoid valves, a Schmidt trigger control scheme was developed that activated when the absolute error between the control signal and actual brake line pressure was greater than $8 \%$.

In order to smoothly blend the mechanical and regenerative braking torques, a control system was created based on finite state machine theory using a programme within Matlab called Stateflow. In this system, inputs such as driver braking demand, electric motor angular speed and state of charge of the ESS are used to optimize the amount of regenerative braking torque delivered by the electric motor, as well as blending in the mechanical braking torque to meet driver demands.

Once the mathematical models were completed, preliminary results could be used to evaluate system performance. For this simulation, the flywheel was accelerated for the first seven seconds, followed by a three second braking demand. During the three second braking demand, a mechanical braking torque control signal was sent to the electro-mechanical brake system. The focus in this paper is the system's response to this signal. The results, showing both the control signal and the actual mechanical braking torque from the simulation, are shown in Figure 7. It can be observed that the virtual prototype can track the control signal within the predefined $8 \%$ relative error with a response time much smaller than 0.6 seconds. These preliminary results demonstrate the functionality of the system to control the mechanical braking torque. However, work can still be done to improve the results recorded.

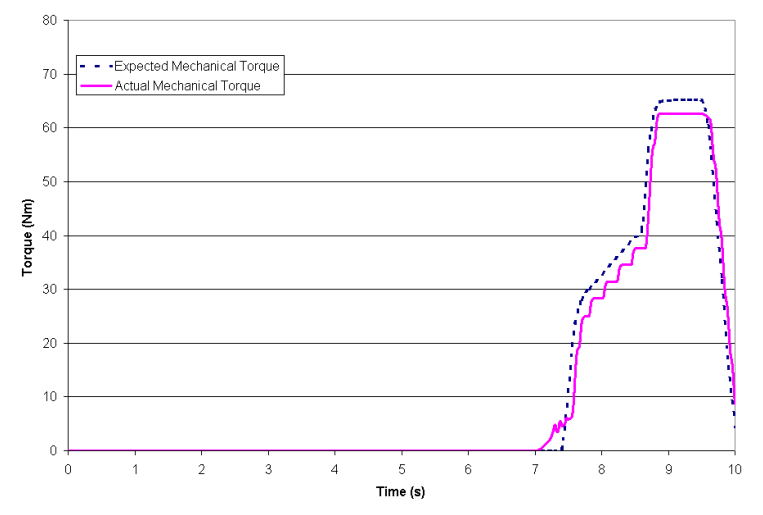

Figure 7: Preliminary simulation results of the electromechanical braking system

\subsection{Physical Prototyping}

To complement the modelling (virtual prototype), a physical prototype of the system was built. This prototype was used to gain physical insight into the system, as well as acting as a quantitative tool to compare real world results with predefined deliverables. The physical prototype also served as a testbench with regards to sourcing physical sensors and solenoid valves that would meet the demands of the system.

The prototype was created as realistically as possible, however some assumptions had to be made during the design. By modifying a standard automotive brake booster into the electro-mechanical brake booster instead of creating a new design, the volumes of the chambers in the brake booster were larger than intended. 
This extra volume affects the air chamber dynamics and causes larger time delays, which decreases the responsiveness of the system. Also, since only one brake caliper was used instead of four, three of the brake valve body ports were plugged, which reduced the length of the brake lines in the system and hence used less brake fluid. This reduction of brake fluid could reduce the damping of the generated braking torque as well as the time constant of the system.

The same control system used in the simulation was used for the prototype testbench. A National Instruments PCI-2024E multifunction I/O device was used along with the real time workshop environment in Matlab to process the sensor readings and control the electro-mechanical brake booster. There is a brake pedal force sensor attached to the brake pedal to determine the driver demand, and a brake line pressure sensor is used as feedback for controlling the valves. The last critical sensor used is a digital encoder attached to the shaft of the electric motor, which measures the angular speed of the shaft and is used to calculate the amount of regenerative braking torque possible.

Once the prototype was operational, physical tests were conducted and the sensor signal results were recorded. The purpose of these first tests was to demonstrate the functionality of the system along with working out any minor issues. One of the first issues encountered with the physical prototype was the noise associated with some of the sensor signals. This noise caused conflicts within the control scheme so a method to filter the sensor signals had to be created. The results from one of the first tests are shown in Figure 8 . The inputs to this system are a physical force applied to the brake pedal, and a brake line pressure control signal. The system controls the actual signal to follow this demand. From the results, it can be observed that the physical system functions in a similar manner to the virtual prototype. The actual brake line pressure tracks the brake line pressure control signal well and physically verifies the feasibility of this system for an automotive application. The only criteria of the project definition that is not fully achieved is the ability to reduce the mechanical braking force to $0 \%$, because some of the pedal force is still transmitted through the brake booster to the master cylinder. However since the transmitted value is small, less than 5\%, it was deemed acceptable.

\subsection{Results}

The virtual and physical prototypes were integrated to further explore the proposed concept. Inputs to the physical prototype were recorded, and used as input to the virtual prototype. This allowed the results to be compared and refinements to be made to the models.

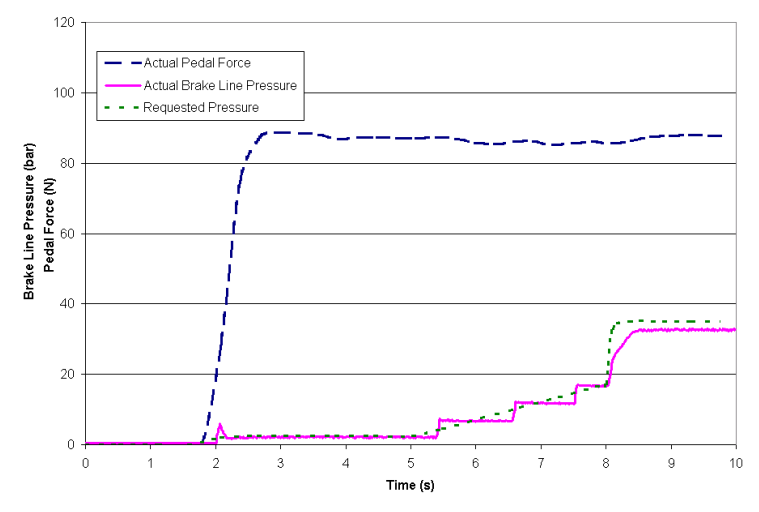

Figure 8: Preliminary results of the prototype electromechanical brake booster

The physical brake pedal force and brake line pressure control signal were recorded and used as inputs to the virtual prototype. The results were then compared and used as a means of refining and validating the mathematic model. This proved very valuable by providing a method to improve the accuracy of the virtual prototype by replacing some of the estimated look-up tables in the simulation with actual physical data. An example of one of these tables converts requested brake line pressure into actual brake line pressure.

Figure 9 shows the results from one of the first comparisons. In this test, a step-like brake pedal force input was created by the driver using the physical system. A control signal was then developed which was tracked by the brake line pressure in both the physical and virtual systems. These brake line pressures were then compared visually to evaluate the two systems. Note that this is the same test shown in Figure 8 with the addition of the virtual prototype results, and enlarged to focus on the brake line pressures.

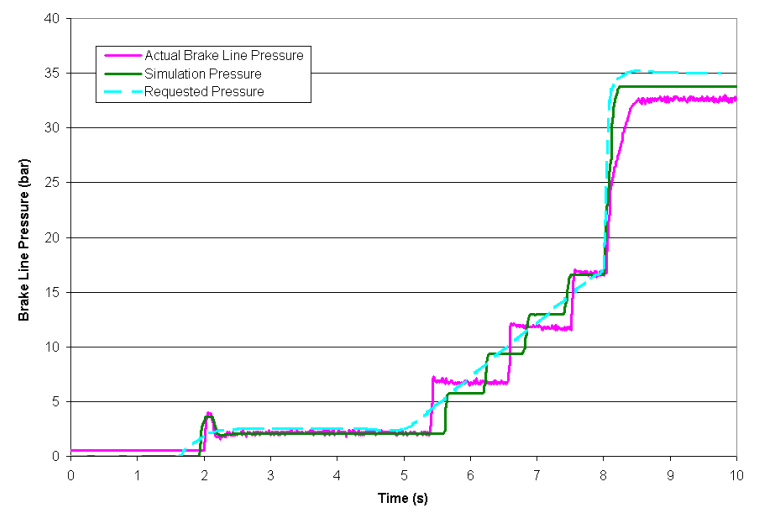

Figure 9: Comparison of the virtual and physical prototypes 
From the results during the ramp section of the control signal (from 5-8 seconds), it was observed that the physical prototype followed the control signal more accurately than the virtual prototype. The inaccuracy of the virtual prototype during the ramp section of the control signal was most likely caused during the conversion of the brake pedal force into the brake line pressure in the simulation. Even though the virtual prototype did not follow the control signal as well, it reacted faster by using four steps during this ramp period where the physical prototype used only three steps. The discrepancy during the step portion of the control signal (from 8-10 seconds) was most likely caused by assuming an infinite volume for the accumulator in the simulation. In reality, a 4 litre accumulator was used, which resulted in a slower change in the physical brake line pressure.

\section{Discussion}

During this design process, creating both virtual and physical prototyping proved to be invaluable. Creating virtual prototypes to determine feasibility of the conceptual design and creating physical prototypes to give physical insight into the concept are both very important. However, using the two prototypes in conjunction proved to be most valuable.

This was best illustrated using the control scheme that governs the electro-mechanical brake booster. Once both prototypes were completed, results from the physical system were used to fine tune the virtual model to respond as realistically to the physical prototype as possible. By doing this, it was then possible to tune the controller of the system using the virtual prototype, which is time efficient and has tight control over the input variables. These control algorithms were then transferred to the physical prototype with a high degree of confidence and they worked as intended.

In conclusion, virtual and physical prototyping are cost and time efficient methods to demonstrate feasibility of a conceptual design, as well as demonstrating that the system can meet the requirements of the project definition. They can be used as a way of solving design problems that may arise during the preliminary design phase and can be easily adapted to test new concepts that may be created. These prototypes can also be used in combination as a powerful tool to validate and develop the system in an efficient and confident manner.

\section{Recommendations}

As the project moves forward, it will be important to continue improving the virtual and physical prototypes. The next step will require incorporating the electric system into the physical prototype to observe how the mechanical and regenerative braking systems interact in real life conditions. Experiments are also scheduled to test proportional control valves as a way of improving both the response and accuracy of the system.

From an overall perspective, the physical prototype was a very useful design tool. It allowed for an understanding of the system that would not have been possible with just the virtual prototype. In many academic situations, physical prototypes are often not completed for either cost or time constraints. However, these prototypes can prove to be great educational resources for students and offer significant added value to the research.

\section{Acknowledgements}

This project was funded by AUTO21, a Network of Centres of Excellence, project E03-RGB. Steve DeMers was supported by an NSERC post graduate scholarship.

\section{References}

[1] J. Lowry, and J. Larminie, Electric Vehicle Technology Explained, John Wiley and Sons, Ltd., West Sussex, England, 2003.

[2] M. H. Westbrook, The Electric and Hybrid Electric Car, The Institution of Electrical Engineers, London, England, 2001.

[3] D. G. Ulman, The Mechanical Design Process, The McGraw-Hill Companies, Inc., New York, NY, 2003.

[4] R. Hodkinson, J. Fenton, Lightweight Electric/Hybrid Vehicle Design, Reed Education and Professional Publishing Ltd., Woburn, MA, 2001.

[5] H.P. Schoner, "Automotive Mechatronics", Control Engineering Practice 12 (2004), Elsevier Ltd., 2004, pp. 13441351.

[6] M.I. Marei, S. J. Samborsky, S. B. Lambert and M. M. A. Salama "On the Characterization of Ultracapacitor Banks Used for HEVs", IEEE Vehicle Power and Propulsion Conference (VPPC), Windsor, UK, 2006.

[7] R. Bosch GmbH, Automotive Handbook, 7th Edition, Robert Bosch GmbH, Plochingen, Germany, 2007.

[8] E. Michels, US Patent 5711204: Electronically controlled brake booster and method of operation, http: / / www . google.com/patents?vid=USPAT5711204 1998.

[9] Y. Kahn, P. Kulkarni, and K. Youcef-Toumi "Modeling, Experimentation and Simulation of a Brake Apply System", Proceedings of the American Control Conference, 1992, pp. 226-230.

[10] Fluid Automation Systems, Kv Flow Factor, http://www.omega.com/techref/pdf/ Solenoid_Valve_Select.pdf 2007. 